\title{
Drop fragment distributions under shear with inertia *
}

\author{
Y. Renardy ${ }^{\mathrm{a}, *}, \quad$ V. Cristini ${ }^{\mathrm{b}}, \quad \mathrm{J} \mathrm{Li}^{\mathrm{c}}$ \\ ${ }^{a}$ Department of Mathematics, Virginia Polytechnic Institute and State University, \\ Blacksburg, VA 24061-0123, USA \\ ${ }^{\mathrm{b}}$ Department of Chemical Engineering and Materials Science, University of \\ Minnesota, Minneapolis, MN 55455-0132, USA \\ ${ }^{\mathrm{c}}$ BP Institute, Bullard Laboratories, University of Cambridge, Madingley Road, \\ Cambridge, CB3 OEZ, UK
}

\begin{abstract}
A numerical investigation is conducted for a drop of viscous liquid, suspended in another liquid of the same density and viscosity, and undergoing breakup due to simple shear. The computational domain is a three-dimensional box, with spatial periodicity on the sides, and no-slip conditions at the top and bottom walls. The full Navier-Stokes equations are solved, with a volume-of-fluid algorithm to track the interface, a continuous surface force or surface stress formulation for modeling interfacial tension, and a semi-implicit Stokes solver to treat order one Reynolds numbers.

We focus on trends for the drop fragment distribution when the flow strength is fixed and the mother drop size is increased. Just above the critical capillary number, the drop evolves to a dumbbell, and breaks according to the end-pinching mechanism. The daughter drops take almost all the mother drop volume; the neck then spawns small moons of less than $1 \%$ the critical volume. When the mother drop size increases, there is repeated end-pinching for the elongated neck region. The fragments consist of large satellites with radii that are two to three times that of the effective neck radius, alternating with small moons. At higher capillary numbers, much of the neck becomes cylindrical and breakup produces large satellites of roughly monodispersed size and volumes in the range $10 \%$ to $17 \%$ of the critical volume, alternating with small moons.

The large mother drops result in daughters that scale with the critical size (and are always the largest fragments produced), together with satellites that also scale with the critical size, and moons which make up a small percentage of the volume. Such scalings allow to predict the fragment size distribution. Our results are consistent with recent experimental observations for Stokes flow. The effect of inertia is a reduction in the size of the fragments.
\end{abstract}

Key words: drop breakup, VOF method

Preprint submitted to Int. J. Multiphase Flow 17 January 2001 


\section{Introduction}

Drop breakup processes provide the mechanisms through which emulsions form, in devices such as rotor-stator mixers (Utracki and Shi, 1992; Kennedy et al., 1994; Cristini et al., 1998; Blawzdziewicz et al., 2000; Cristini et al., 2001a,b). Past studies have concerned Stokes flows; however, commercial devices may operate under speeds where inertial effects enter. In this paper, we focus on order one Reynolds numbers.

The study of fragmentation contributes to an understanding of the manner in which these shearing devices produce droplets and the drop size distributions that result from the sequence of events. Eventually, the flow equilibrates with drop sizes that depend on physical properties and conditions of shear. At that stage, the drop size distribution affects the rheological properties of the final emulsions. This work applies to dilute suspensions for which coalescence is negligible, and equilibrium drop sizes are determined by breakup.

The evolution of a drop under simple shear is a three-dimensional problem, and requires a direct numerical simulation with the full governing equations. Section 2 summarizes our numerical algorithm, which is a finite difference scheme on a Cartesian grid. Drop deformation depends on the viscosity ratio, capillary number and Reynolds number. The effects of these parameters up to the formation of the first daughter drops are given in Guido and Villone (1998); Cristini (2000); Renardy and Cristini (2001, 2000). We focus on the case of equal viscosities and densities for the drop and matrix liquids, and investigate neck breakup by keeping the flow strength the same and increasing the drop size.

When the viscosities, surface tension, and flow strength are fixed, the variation

\footnotetext{
ऋ This research was sponsored by NSF-INT 9815106, NSF-DMS SCREMS, and utilized the NCSA SGI Origin 2000. Acknowledgement is made to the donors of The Petroleum Research Fund, administered by the ACS, for partial support of this research.

* Corresponding author.

Email addresses: renardyy@math.vt.edu (Y. Renardy), cristini@cems.umn.edu (V. Cristini), j1305@eng.cam.ac.uk (J. Li).

$U R L$ : www.math.vt.edu/people/renardyy (Y. Renardy).
} 
in mother drop size yields the relationship

$$
R e / C a^{2}=K
$$

where $K=\rho \sigma^{2} /\left(\mu^{3} \dot{\gamma}\right)$ is a constant. Here, $\dot{\gamma}$ is the imposed shear rate, $C a=$ $\mu \dot{\gamma} a / \sigma$ is the capillary number, where $\mu$ is the viscosity, $a$ is the initial radius of the drop, and $\sigma$ is interfacial tension, $R e=\rho \dot{\gamma} a^{2} / \mu$ is Reynolds number on the drop scale. We define a daughter capillary number and Reynolds number,

$$
C a_{D}=(D / a) C a, \quad R e_{D}=\operatorname{Re}\left(D^{2} / a^{2}\right)
$$

where $D$ is the daughter drop radius ( $D^{3}<a^{3} / 2$ from volume conservation). We shall find in Section 3 that there is a self-similar behavior for the size of the fragments. The fragments all scale with the critical condition, so that an important parameter is the critical capillary number $C a_{c}$, above which the drop breaks up; the corresponding critical radius is denoted $a_{c}$. The maximum stable drop size is attained at $C a_{c}$.

Above the critical capillary number, the drop elongates, bulbs form at the ends, and these produce the largest daughter drops. For Stokes flow, the volume of the first daughter drops becomes insensitive to the mother drop size when the capillary number increases (Cristini, 2000). It saturates at a value (weakly dependent on the fluid viscosities) roughly equal to $70 \%$ of the critical volume $V_{c}=(4 / 3) \pi a_{c}^{3}$, corresponding to a daughter capillary number $C a_{D} \approx 0.9 C a_{c}$. A similar scaling holds with inertia, together with a reduction of the daughter drop size (Renardy and Cristini, 2000). As the Reynolds number increases, the flow within the drop becomes more complicated, the dumbbell aligns at a higher angle of tilt before breakup, and this apparently contributes to the reduction in size of the daughter drops.

When the first daughter drops pinch off, the neck is fully elongated. As the capillary number increases, more volume goes into the neck, which becomes almost perfectly cylindrical for much of its length. The ends spawn drops, and then the remaining central portion undergoes simultaneous capillary breakup. This final stage is close to, but not exactly, an axisymmetric jet undergoing capillary jet breakup, which is an energy minimization process that minimizes surface area by breaking into drops. The wavelength of maximum growth rate for an inviscid jet (Rayleigh, 1900) yields a drop radius of 1.87 times the jet radius. The numerical work of Tjahjadi et al. (1992) on capillary breakup of a filament in a quiescent viscous fluid yields drops with twice the jet radius. These works are similar to our situation, with one difference being that our filament is aligned at an angle to the flow; the angle of tilt is higher at the larger Reynolds numbers. In Section 3, we find the drops to be two to three times an 'effective' neck radius for capillary numbers where repeated end-pinching occurs. 


\section{Numerical algorithm}

\subsection{Formulation}

Our code SURFER++ is composed of three parts: a second-order volumeof-fluid VOF method to track the interface (Scardovelli and Zaleski, 1999; Gueyffier et al., 1999), a projection method to solve the Navier-Stokes equations on the MAC grid, and finally, a continuum method for modeling the interfacial tension. The details of the original code SURFER are given in Li (1995); Gueyffier et al. (1999), and new capabilities of SURFER++ are described in Li et al. (1998); Li and Renardy (1999), and Li et al. (2000): a summary is provided below.

The density $\rho$ and the viscosity $\mu$ of each fluid is a constant in each fluid. A concentration (or color) function $C$ is used to track the interface:

$$
C(\mathbf{x})=\left\{\begin{array}{l}
1 \text { fluid } 1 \\
0 \text { fluid } 2
\end{array}\right.
$$

This concentration function is transported by the velocity field $\mathbf{u}$. The fluids are incompressible: $\nabla \cdot \mathbf{u}=0$, and governed by the Navier-Stokes equation:

$$
\rho\left(\frac{\partial \mathbf{u}}{\partial t}+\mathbf{u} \cdot \nabla \mathbf{u}\right)=-\nabla p+\nabla \cdot \mu \mathbf{S}+\mathbf{F}
$$

where $\mathbf{S}$ is the viscous stress tensor. In the VOF method, the interfacial tension condition across the interface is expressed as a body force over the mesh cells which contain the interface. We implement the continuous surface stress (CSS) formulation (Lafaurie et al., 1994), in which $\mathbf{F}_{s}=\nabla \cdot \mathbf{T}=\sigma \delta_{\mathbf{S}} \kappa \mathbf{n}_{\mathbf{S}}$ and $\mathbf{T}=\left[\left(\mathbf{1}-\mathbf{n}_{\mathbf{S}} \otimes \mathbf{n}_{\mathbf{S}}\right) \sigma \delta_{\mathbf{S}}\right]$. No mollification is used on the color function.

The temporal discretization is performed with Chorin's projection method, which decouples the pressure equation. The semi-implicit Stokes solver described in Li and Renardy (1999); Li et al. (1998), and Li et al. (2000) is used. The spatial discretization is a Cartesian mesh of rectangular cells. It is a finite difference mesh known as the MAC grid. The density and viscosity for each cell are given by $\rho=C \rho_{1}+(1-C) \rho_{2}$ and $\mu=C \mu_{1}+(1-C) \mu_{2}$, where subscripts refer to fluids 1 and 2 .

A piecewise linear interface calculation (PLIC) method is used to reconstruct the interface position. The approximate normal $\mathbf{n}$ to the interface in each cell is equal to the discrete gradient of the volume fraction field: $\mathbf{n}=\nabla^{h} C /\left|\nabla^{h} C\right|$. The final step of the VoF method is to evolve the volume fraction field $C$. At the nth timestep, the interface is reconstructed, the velocity at the interface 


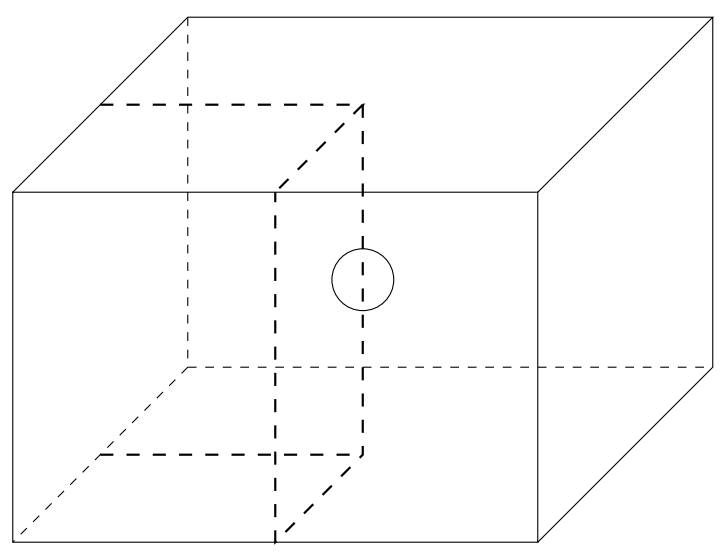

(a)
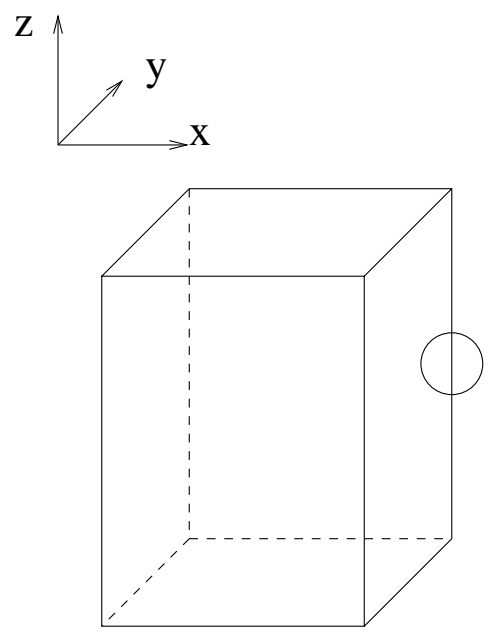

(b)

Fig. 1. (a) Full domain of computation. (b) Domain of computation by using symmetries.

is interpolated linearly and then the new interface position for the $(n+1)$ th timestep is calculated via a Lagrangian method: $\mathbf{x}^{n+1}=\mathbf{x}^{n}+\mathbf{u}(\Delta t)$. The code is parallelized; data on the scalability and timings are given in Renardy and Li (2000).

\subsection{Implementation of symmetries in SURFER++}

We have implemented the anti-symmetry condition in the x-direction and the symmetry condition in the y-direction. The difficulty in implementing these conditions originates from the MAC staggered mesh, for which we must distinguish three different cases for the velocity components $u, v$ and $w$. This implementation results in a four-fold reduction of machine memory and a reduction of $\mathrm{CPU}$ time of at least four times.

For the drop deformation and break-up in simple shear flow, we used periodic boundary condtions in both $\mathrm{x}$ and $\mathrm{y}$ directions and the no-slip condition at the top and bottom walls. We find that at our flow conditions, the solution displays symmetries. As shown in Fig. 1 (a), suppose the drop is initially at the center of the computational box; by using the anti-symmetrical condition in $\mathrm{x}$ and symmetrical condition in $\mathrm{y}$, we reduce the domain for the computation to one quarter of the original domain. This is illustrated in Fig. 1 (b). We can therefore use one quarter of memory of the previous computation. Moreover, we also reduce at least four time the CPU, as the CPU increases more quickly than a linear dependance on the mesh size.

Implementation of the symmetry in the $\mathrm{y}$ direction is trivial. Here, we show 


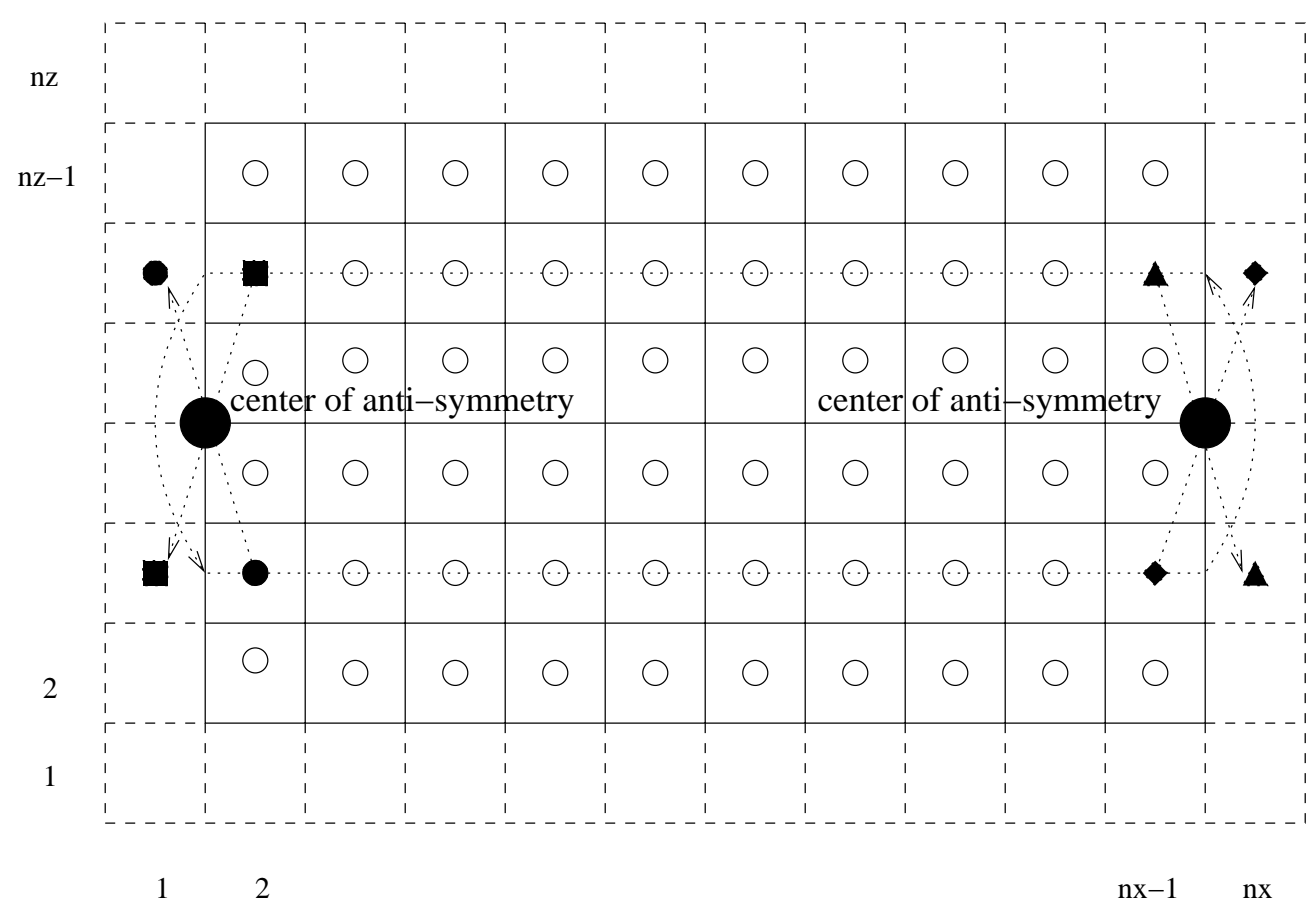

Fig. 2. Symmetry condition for constant variables: volume fraction $C$, pressure $p$ and velocity component $v$.

only how to implement the anti-symmetrical condition in the $\mathrm{x}$ direction. Due to the staggered mesh, the variables are defined on different locations. We therefore discuss three different cases. We begin with the the constant variables: volume fraction $C$, pressure $p$ and velocity component $v$. Consider an $\mathrm{X}-\mathrm{Z}$ plane: these variables are defined at the centers of the cells, as shown in Fig. 2. The phyiscal domain contains the cells from $(2,2)$ to $\left(n_{x}-1, n_{z}-1\right)$ (represented by solid lines). The cells on the border (represented by dashed lines) are ghost cells. They are useful for implementing boundary conditions. Therefore, the declarations for these variables in a Fortran program are $c c\left(n_{x}, n_{z}\right), p\left(n_{x}, n_{z}\right)$ and $v\left(n_{x}, n_{z}\right)$. The two centers of anti-symmetry are at the centers of the right and left borders of the physical domain (represented by two large black circles). Whenever we need to impose the antisymmetrical condition in the explicit scheme, we just copy the appropriate values to the ghost cells: see Fig. 2, for instance, $c c(1, k)=c c\left(2, n_{z}-k+1\right)$ and $c c\left(n_{x}, k\right)=c c\left(n_{x}-1, n_{z}-k+1\right)$. On the other hand, there is a complication in the implementation of the semi-implicit scheme for $v$. As shown in Fig. 2, the row $k$ and the row $n_{z}-k+1$ form a periodic chain and must be solved together. The algorithm for solving this periodic chain is identical to what is already in SURFER++, but it has been rewritten in a more compact and readable form.

The velocity component $u$ is defined at the centers of the vertical borders of each cell. In the physical domain, it is defined from column 2 to the column $n_{x}$. As shown in Fig. 3, we require a column of ghost values for $u\left(n_{x}, *\right)$. 


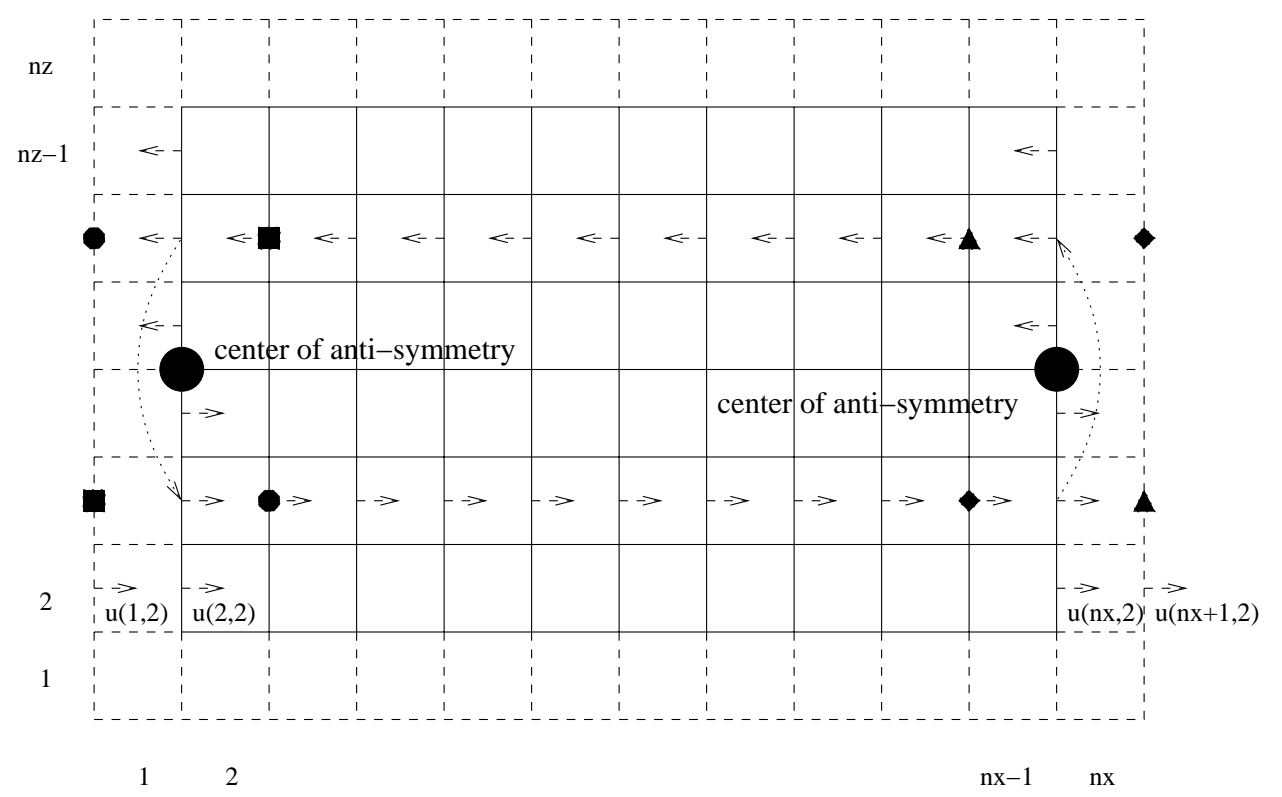

Fig. 3. Implementation of anti-symmetric condition for velocity component $u$.

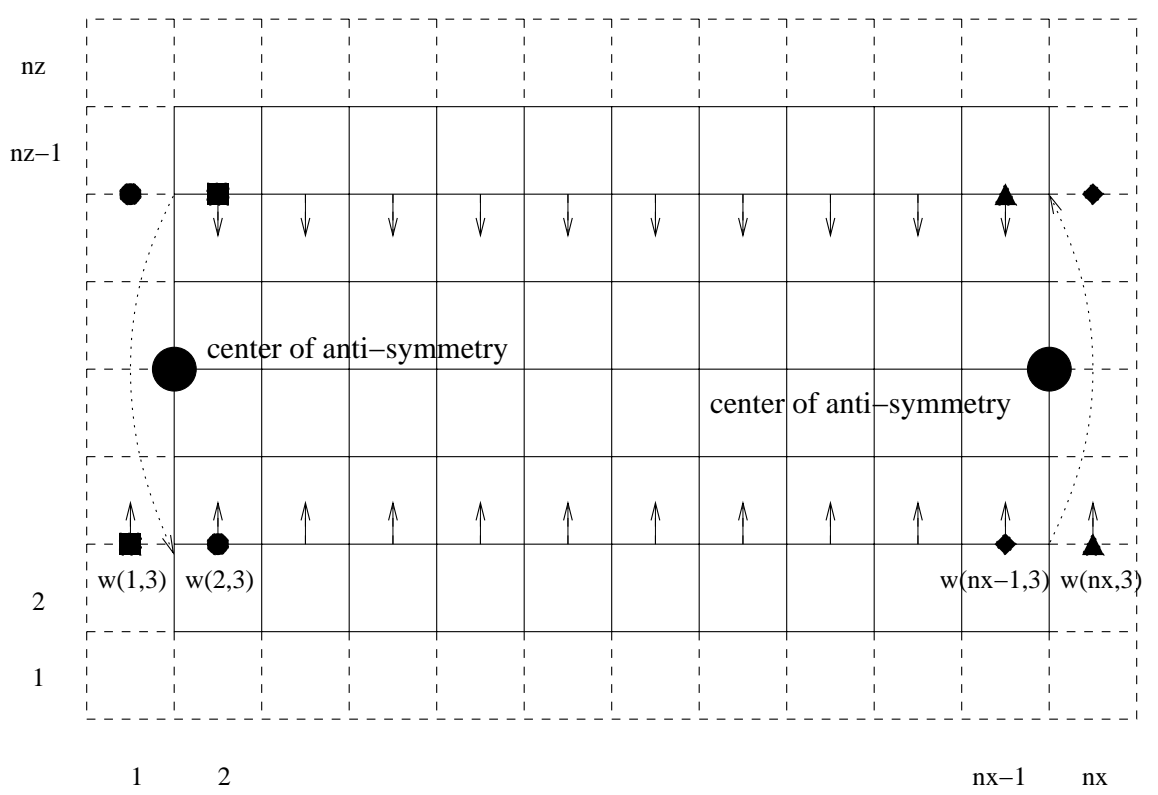

Fig. 4. Implementation of anti-symmetric condition for velocity component $w$.

Therefore, the declaration for $u$ is different from the other variables: it is $u\left(n_{x}+1, n_{z}\right)$. For the explicit scheme, we impose the boundary condition $u(1, k)=-u\left(3, n_{z}-k+1\right)$ and $u\left(n_{x}+1, k\right)=-u\left(n_{x}-1, n_{z}-k+1\right)$. The complication for implementing the semi-implicit scheme is as mentioned above.

The same idea applies to the velocity component $w$ (see Fig. 4): its declaration is $w\left(n_{x}, n_{z}\right)$. We impose the boundary condition $w(1, k)=-w\left(2, n_{z}-k+2\right)$ and $w\left(n_{x}, k\right)=-w\left(n_{x}-1, n_{z}-k+2\right)$. We also follow the above procedure to implement the semi-implicit scheme. 


\subsection{Computational accuracy and efficiency}

An investigation of the effect of the computational domain size, time step and mesh was conducted in Renardy and Cristini (2000) with regard to the volume of the first daughter drops. There, the computational box $L_{x} \times 0.5 \times 1$, with initial drop radius $a=0.125$, time steps $\Delta t=10^{-3} \dot{\gamma}^{-1}$ was sufficient. Note that in Renardy and Cristini (2000), the domain was doubled in both the y and $\mathrm{z}$ directions, and timesteps were halved, with little effect on the volumes of the first daughter drops. The mesh is $N_{x} \times N_{y} \times N_{z}$ and each discretized cell size is

$$
\Delta x=L_{x} / N_{x}, \Delta y=L_{y} / N_{y}, \Delta z=L_{z} / N_{z}
$$

where $L_{y}=0.5, L_{z}=1$. For $L_{x}=2.5$, and mesh $\Delta x=1 / 96,1000$ timesteps require 1.5 hours with 64 processors on the Origin 2000 at NCSA. For $L_{x}=$ 4 and mesh $\Delta x=1 / 128,1000$ timesteps require 13 to 14.5 hours with 8 processors on Origin 2200, or 10 hours with 32 processors on the NCSA Origin 2000 .

\subsubsection{Stationary shapes}

The results of our code are compared with those of the boundary integral code of Cristini et al. $(1998,2001 \mathrm{a})$ for Stokes flow at $C a=0.35$. Their steady-state solution is shown in figure 5 (a). Our code is run at finite Reynolds numbers, and an extrapolation is expected to give the Stokes flow result. At $C a=0.35$, the critical Reynolds number is between 0.2 and 0.4 .

We compute the steady-state solution by advecting the interface for 20 timesteps for every velocity field calculation. This is repeated until steady-state is reached. This method gives the steady-state solution by economizing the CPU time. In tables 1 - 4 , the steady state values for $L / a$ are shown for various computational conditions. Table 1 shows that hydrodynamic interactions with neighboring drops due to spatial periodicity in the y-direction enhances drop deformation.

Table 2 shows refinements in timesteps and we will use $\dot{\gamma} \Delta t=10^{-4}$ for table 3 .

In table 3, the first entry is for the smallest box with respect to the drop radius. When the walls are taken apart and the box widened in the other directions, $L / a$ lowers in value toward the 1.7171 value of the boundary integral code. Next, the Reynolds number is reduced by half. Linear extrapolation in the Reynolds number gives $L / a=1.75$ for Stokes flow, which is $2 \%$ different from that of the boundary integral code. SURFER++ is second-order in space. Thus, the errors are proportional to $(\Delta x)^{2}$. We use the third and fourth rows 

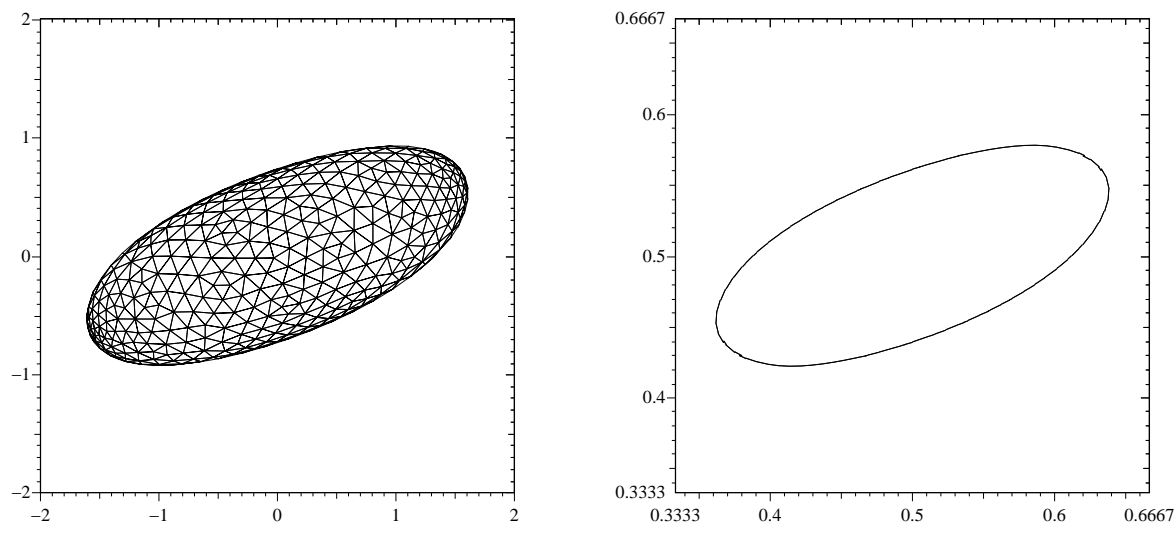

Fig. 5. (a) Steady-state $L / a=1.7171$. (b) Computational box $12 a \times 12 a \times 12 a$, $a=0.08 \dot{3}$, mesh $\Delta x=\Delta y=\Delta z=3 a / 32$, timestep $\dot{\gamma} \Delta t=10^{-4}, R e=0.03125$. Steady-state $L / a=1.760$.

Table 1

\begin{tabular}{||ccccc||}
\hline Comp. Box & Mesh $\Delta x$ & $\Delta t$ & $R e$ & $L / a$ \\
\hline $8 a \times 4 a \times 8 a$ & $a / 8$ & $10^{-3}$ & 0.0625 & 1.935 \\
$8 a \times 8 a \times 8 a$ & $a / 8$ & $10^{-3}$ & 0.0625 & 1.90 \\
\hline
\end{tabular}

Steady-state drop data computed with CSF. $C a=0.35$. Variation with depth in y-direction

Table 2

\begin{tabular}{||ccccc||}
\hline Comp. Box & Mesh $\Delta x$ & $\Delta t$ & $R e$ & $L / a$ \\
\hline $8 a \times 4 a \times 8 a$ & $a / 8$ & $10^{-3}$ & 0.0625 & 1.94 \\
$8 a \times 4 a \times 8 a$ & $a / 8$ & $10^{-4}$ & 0.0625 & 1.87 \\
$8 a \times 4 a \times 8 a$ & $a / 8$ & $0.5 \times 10^{-4}$ & 0.0625 & 1.86 \\
\hline
\end{tabular}

Steady-state drop data computed with CSF. $C a=0.35$. Variation with timesteps.

of the table to calculate the difference $E_{1}=1.79-1.72=C_{1}(a / 8)^{2}$ and $E_{2}=1.766-1.717=C_{2}(3 a / 32)^{2}$. We find that $C_{1}$ and $C_{2}$ are approximately 5 . To reduce the difference with the boundary integral code to $0.5 \%$ would require a mesh of $a / 22$. The calculation in the last row of the table already requires 35 to 40 hours $\mathrm{CPU}$ on 8 processors. 


\begin{tabular}{||ccccc||}
\hline Comp. Box & Mesh $\Delta x$ & $\Delta t$ & $R e$ & $L / a$ \\
\hline $4 a \times 4 a \times 4 a$ & $a / 8$ & $10^{-4}$ & 0.0625 & 1.87 \\
$8 a \times 8 a \times 8 a$ & $a / 8$ & $10^{-4}$ & 0.0625 & 1.80 \\
$12 a \times 12 a \times 12 a$ & $a / 8$ & $10^{-4}$ & 0.0625 & 1.79 \\
\hline $12 a \times 12 a \times 12 a$ & $3 a / 32$ & $10^{-4}$ & 0.0625 & 1.766 \\
$12 a \times 12 a \times 12 a$ & $3 a / 32$ & $10^{-4}$ & 0.03125 & 1.760 \\
\hline
\end{tabular}

Table 3

Steady-state drop data computed with CSF. $C a=0.35$. Variation with spatial refinement and Reynolds number.

\begin{tabular}{|cccc||}
\hline Comp. Box & Mesh $\Delta x$ & $R e$ & $L / a$ \\
\hline $8 a \times 8 a \times 8 a$ & $a / 16$ & 0.0625 & 1.80 mollified \\
$8 a \times 8 a \times 8 a$ & $a / 20$ & 0.03125 & 1.81 not mollified \\
$12 a \times 12 a \times 12 a$ & $3 a / 32$ & 0.03125 & 1.80 mollified \\
$12 a \times 12 a \times 12 a$ & $3 a / 32$ & 0.03125 & 1.787 not mollified \\
$12 a \times 12 a \times 12 a$ & $3 a / 32$ & 0.015625 & 1.80 mollified \\
\hline
\end{tabular}

Table 4

Steady-state drop data computed with CSS. $C a=0.35, \Delta t=10^{-4}$.

A comparison of the Continuous Surface Force (CSF) algorithm with the Continuous Surface Stress (CSS) algorithm with smoothing and without smoothing is done for the last row of table 3. (For details of the CSF and CSS algorithms, we refer the reader to Renardy et al. (2000). Note that CSF requires smoothing in order to be numerically stable, while CSS does not.) The computational box is $12 a \times 12 a \times 12 a$, the mesh is $3 a / 32, R e=0.03125$, timesteps are refined to $\Delta t=0.5 \times 10^{-4}$. The smoothed CSS result settles to $L / a=1.795$, and the unsmoothed to $L / a=1.787$ (see table 4 ). We conclude that if we have sufficient spatial and temporal resolution, no smoothing is preferred. The transient evolution calculated with CSS with no smoothing is shown in figure 6 at $R e=0.03125$, for the ratio of the half-length to radius, vs capillary time $t_{c a}$. For shear rate 1,1 unit of capillary time is equivalent to 0.35 s. Each capillary time requires 3 hours 10 minutes of CPU on 8 processors of Origin 2200. The simulation was stopped at 18s, and compared with the result from the boundary integral method of Cristini (2000). The effect of inertia, hydrodynamic interactions with neighboring drops, and finite distance to walls, in addition to spatial discretization, contribute to the difference with the boundary integral method. 


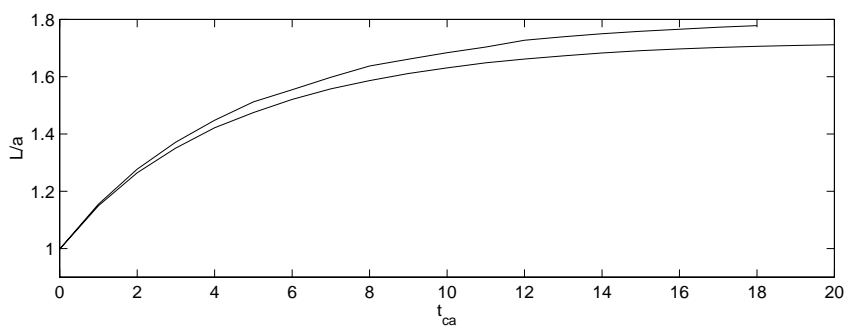

Fig. 6. $L$ denotes half-length of drop. The upper curve shows the transient to $18 \mathrm{~s}$ using the CSS algorithm with no mollification, $R e=0.03125, C a=0.35$, computational box $12 a \times 12 a \times 12 a$, mesh $\Delta x=\Delta y=\Delta z=3 a / 32$, timestep $\Delta t=10^{-4}$, $R e=0.03125$. The lower curve is from the boundary integral code of Cristini (2000).

\subsubsection{Breakup fragments}

The aim of this paper is to extend the results of Renardy and Cristini (2000) which show that the first daughter drops scale with the critical volume. We begin with the study of $R e=12, C a=0.175$, and compare the CSF and CSS algorithms; the results are shown in figure 7 for a rough mesh. This compares the drop evolution for at $18,22,24 \mathrm{~s}$, with timesteps $\Delta t=10^{-3} s$. The CSF algorithm is used in (a) with spatial mesh $128 \times 32 \times 64$ on a computational domain $2.0 \times 0.5 \times 1.0$. In (b), the CSS algorithm is used with spatial mesh $160 \times 32 \times 64$ on a computational domain $2.5 \times 0.5 \times 1.0$. In comparison, the CSF drop has already broken at 18s. After breakup, the ratios of daughter to mother radii for the CSF calculation are, from the first daughter drop toward the center drop, $D / a=0.73$, three moons, 0.37 , moon, then center drop 0.43 . We shall refer to drops with

$$
D / a<0.3
$$

as moons. For the CSS result, the central drop evolves to steady state, and we have $D / a=0.71$, two very close moons, 0.37 , moon, center drop 0.49 . The smoothing evidently speeds up the breakup, and yields a jaggedness in the final pinch-offs which is less pronounced in the case when smoothing is not used. Table 5 shows the daughter capillary numbers and Reynolds numbers. The first daughters differ by $3 \%$ in $D / a$ which is equivalent to $9 \%$ in the ratio of daughter to critical volume,

$$
V_{D} / V_{c}=\left(\left(\frac{D}{a}\right)\left(\frac{C a}{C a_{c}}\right)\right)^{3}
$$

With mesh refinement, the values for CSF and CSS approach each other. For example, the CSF with $192 \times 32 \times 128$ on a computational domain $3.0 \times 0.5 \times$ 1.0 gives $D / a=0.732$, the CSS with mollification on $480 \times 96 \times 192$ on a computational domain $2.5 \times 0.5 \times 1$ gives 0.726 , and without mollification, 0.718. Thus, the main reason for the difference in figure 7 and table 5 is the 


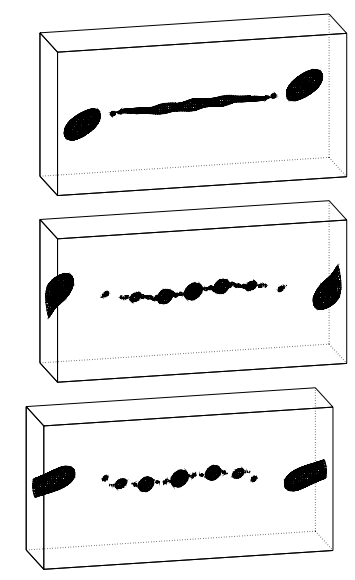

(a)

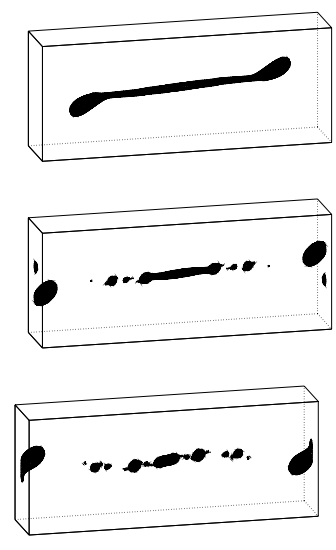

(b)

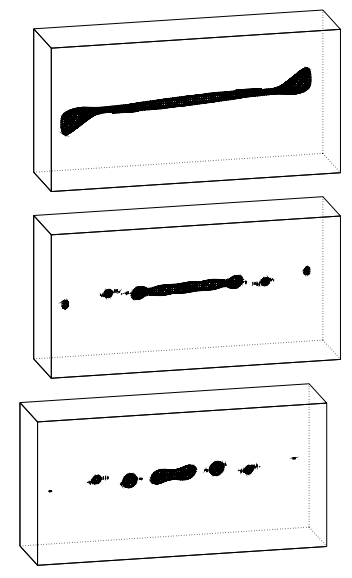

(c)

Fig. 7. $R e=12, C a=0.175=1.14 C a_{c}$, sequence of evolution at $\mathrm{t}=18,22,24 \mathrm{~s}$. (a) CSF, (b) CSS with no mollification. In (a), at $\mathrm{t}=22 \mathrm{~s}$, the first daughters have exited the domain and merged with the first daughters of the next mother drop because of spatial periodicity in the x-direction. (c) Computational domain $2.0 \times 0.5 \times 1.0$. Drops are erased as they exit the domain.

\begin{tabular}{||c|ccc|ccc|ccc||}
\hline & CSF & & & CSS & & & CSS erased & & \\
Drop & $D / a$ & $C a_{D}$ & $R e_{D}$ & $D / a$ & $C a_{D}$ & $R e_{D}$ & $D / a$ & $C a_{D}$ & $R e_{D}$ \\
\hline 1 & 0.73 & 0.13 & 6.47 & 0.71 & 0.13 & 6.11 & 0.71 & 0.13 & 6.12 \\
2 & 0.37 & 0.07 & 1.64 & 0.37 & 0.07 & 1.67 & 0.36 & 0.06 & 1.51 \\
center 3 & 0.43 & 0.08 & 2.18 & 0.49 & 0.09 & 2.90 & 0.55 & 0.10 & 3.63 \\
4 & 0.37 & 0.07 & 1.64 & 0.37 & 0.07 & 1.67 & 0.36 & 0.06 & 1.51 \\
5 & 0.73 & 0.13 & 6.47 & 0.71 & 0.13 & 6.11 & 0.71 & 0.13 & 6.12 \\
\hline
\end{tabular}

Table 5

$R e=12, C a / C a_{c}=1.14$. Drop distribution computed with the CSF algorithm and CSS algorithm with no smoothing. Ratio of daughter radius to mother radius $D / a$, daughter capillary number $C a_{D}$, and daughter Reynolds number. Mesh $\Delta x=\Delta y=$ $\Delta z=1 / 64$. Total number of main drops, excluding moons, is 5 .

presence of smoothing.

During the course of the computation, the neighboring first daughter drops enter into the computational domain because of spatial periodicity in the $\mathrm{x}$ direction, and undergo interactions with other satellite drops as the breakup progresses. In order to prevent this interaction, drops will be erased as they exit the left and right hand boundaries. Figure 7 (c) shows the effect of erasure on the simulations of (a) and (b). In (c), we can see parts of the first daughters erased at $18 \mathrm{~s}$. The drop distribution is also shown in table 5 . The 
effect of erasure is less than $1 \%$ on the $D / a$ for the first daughters, but is more on the satellite drops. Hydrodynamic interactions among the drops at close proximity in (a) and (b) have been decreased in (c). In conclusion, the CSS algorithm with no mollification will be used in section 3. Moreover, we shall erase the drops as they exit the computational domain in order to optimize computational effort and decrease hydrodynamic interactions with spatially periodic neighboring drops.

Next, we turn our attention to the temporal refinement. Figure 8 shows the breakup sequence for two spatial refinements, conducted with the CSS algorithm with no mollification, and with drops erased as they exit the domain. These are snapshots taken from the top of the computational domain. These simulations are at $\Delta t=10^{-3}$. These were repeated at one tenth of this timestep and the results are identical. Thus, $\Delta t=10^{-3}$ will be used.

Finally, we examine the spatial refinement. Figure 8 shows that the time to breakup of the first daughters increases with spatial refinement. Secondly, the small satellites which come off of the neck just after the first daughters pinch off are on the scale of the mesh. Thirdly, the 1/96 mesh results shown in (b) are not physically acceptable because the center drops split without a moon in between. Past results on breakup have always resulted in small moons between larger drops. Fourthly, the number of main drops, disregarding small moons, is 5 for the 1/64 mesh and 6 for the 1/96 mesh. The drop data for the $1 / 64$ case is the last columns of table 5 . The $1 / 96$ case gives $D / a=0.72$ for the first daughters, then toward the center, moon, $0.34,0.43$. Clearly, more spatial refinement is required.

Figure 9 shows results for $1 / 128,1 / 160$, and $1 / 192$ meshes. The drop data are given in table 6 . First, the singularity which arises after the first daughters pinch off affects the resolution of the subsequent small drops (Papageorgiou, 1995; Eggers, 1995; Lister and Stone, 1998). For example, for the 1/192 mesh, there are a couple of tiny moons. In figure $9(\mathrm{c})$, the last picture at $\mathrm{t}=29 \mathrm{~s}$ shows the largest of these moons, drop 2, moon with $D / a=0.09, C a_{D}=$ $0.016, R e_{D}=0.09$, and center drop 3. The central three-drop structure in this picture is the most realistic one, being the last of the end-pinching sequence, evolving into the familiar dumbbell ends with more volume in the dumbells than in the neck. Secondly, as the mesh is refined, the time to breakup for the final satellites lengthens with better resolution of the neck region. The 1/192 case takes 33 hours CPU on 8 processors of Origin2200 per 1000 timesteps (1s), totalling 40 days CPU up to the stage shown in the figure. When the full $3 \mathrm{D}$ code is re-structured to take into account the symmetry in the y-direction and anti-symmetry in the $\mathrm{x}-\mathrm{z}$ plane, then 1 s takes approximately 11 hours CPU per 1s. 
(a)
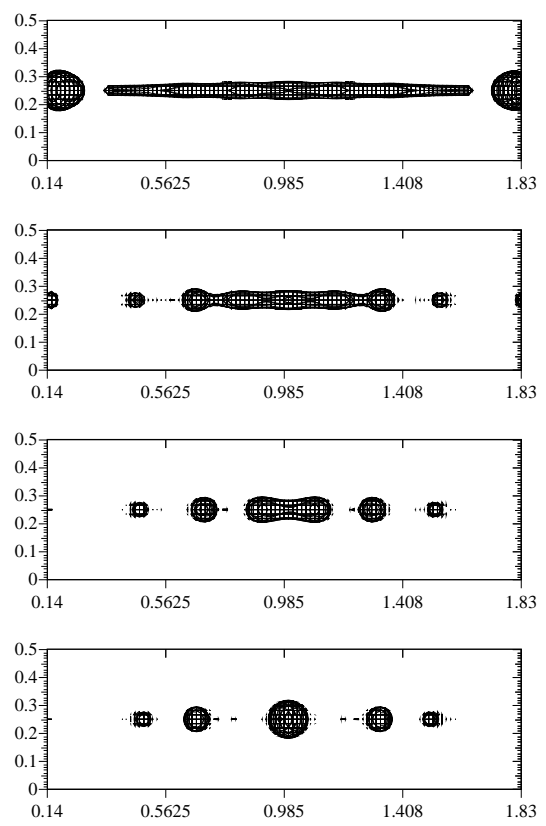

(b)
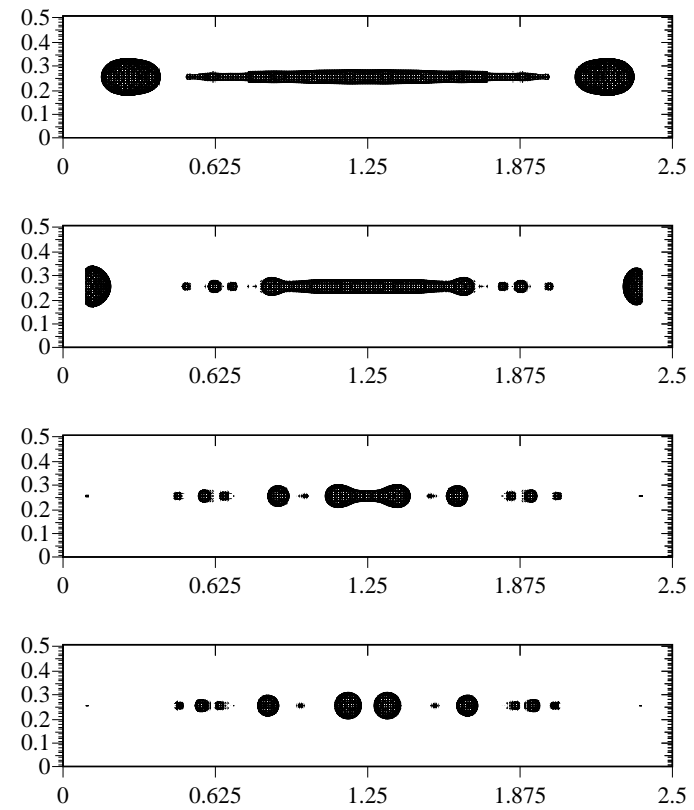

Fig. 8. $R e=12, C a=0.175=1.14 C a_{c}$, top view. This is the breakup sequence for the parameters of figure 2(b) of Renardy and Cristini (2000) for computational domains (a) $2 \times 0.5 \times 1$, mesh $\Delta x=\Delta y=\Delta z=1 / 64$. (b) $2.5 \times 0.5 \times 1$. mesh 1/96. (a) $\mathrm{t}=19$ (drop 1 detached), $\mathrm{t}=22$ (moon detached), 24 (drop 2 detached, center evolves to dumbbell), 26s (center retracts to single drop). (b) $t=20$ (drop 1 detached), 22 ( 3 moons detached), 25 (3 moons, drop 2, moon, detached), 27s (center drops detached).

The least spatially refined case already captures the size of the first daughter drops, which are $54 \%$ of the critical drop volume, together with the gross features of the breakup. Table 6 shows that the volumes of the neck drops are $10 \%$ to $15 \%$ of the critical drop volume. The convergence for the smaller drops with $D / a<0.3$ requires a much finer mesh. In particular, the moons which are spawned from the neck immediately after the first daughter drops detach are extremely small. On the rough mesh, they are therefore resolved to yield a larger volume, giving less volume to the main drops. Comparing the $1 / 160$ and 1/192 meshes, we see that the resolution of the main drops from the neck is achieved at the 1/160 case. At a higher capillary number, more of the volume goes into the neck and its breakup will approach that of a filament. In this case, sufficient refinement is not feasible without the use of an adaptive grid. However, the results in table 6 indicate that the values for drops 2 and 3 corresponding to resolutions $\Delta x=1 / 128,1 / 160,1 / 192$ roughly converge as 


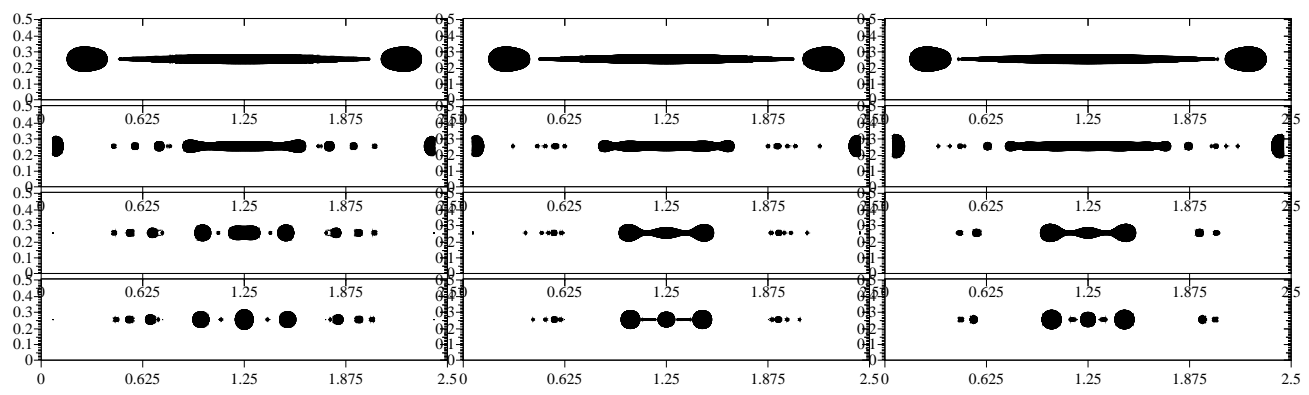

(a)

(b)

(c)

Fig. 9. $R e=12, C a=0.175=1.14 C a_{c}$, top view. This is the breakup sequence for the parameters of figure 2(b) of Renardy and Cristini (2000) for computational domain $2.5 \times 0.5 \times 1$. (a) mesh 1/128. $\mathrm{t}=20$ (drop 1 detached), 23 ( 3 moons), 26 (drop 2), 27s. (b) mesh 1/160. t=20 (drop 1 detached), 23,26,27s. (c) mesh 1/192. $\mathrm{t}=20,23,28,29 \mathrm{~s}$.

\begin{tabular}{||c|cccc|ccccc|cccc||}
\hline $\begin{array}{c}\text { Drop } \\
\Delta x\end{array}$ & $\mathrm{D} / \mathrm{a}$ & & & & \multicolumn{1}{l|}{$C a_{D}$} & & & & \multicolumn{2}{l||}{$R e_{D}$} & & \\
\hline & $1 / 64$ & $1 / 128$ & $1 / 160$ & $1 / 192$ & $1 / 64$ & $1 / 128$ & $1 / 160$ & $1 / 192$ & $1 / 64$ & $1 / 128$ & $1 / 160$ & $1 / 192$ \\
\hline 1 & 0.71 & 0.72 & 0.72 & 0.72 & 0.13 & 0.13 & 0.13 & 0.13 & 6.10 & 6.18 & 6.19 & 6.19 \\
2 & 0.36 & 0.39 & 0.45 & 0.46 & 0.06 & 0.07 & 0.08 & 0.08 & 1.54 & 1.84 & 2.44 & 2.58 \\
3 & 0.55 & 0.47 & 0.40 & 0.35 & 0.10 & 0.08 & 0.07 & 0.06 & 3.62 & 2.63 & 1.89 & 1.50 \\
4 & 0.36 & 0.39 & 0.45 & 0.46 & 0.06 & 0.07 & 0.08 & 0.08 & 1.54 & 1.84 & 2.44 & 2.58 \\
5 & 0.71 & 0.72 & 0.72 & 0.72 & 0.13 & 0.13 & 0.13 & 0.13 & 6.10 & 6.18 & 6.19 & 6.19 \\
\hline
\end{tabular}

Table 6

$R e=12, C a / C a_{c}=1.14$. Trends with spatial refinement. Mesh is given by $\Delta x=$ $\Delta y=\Delta z$ in each direction. Drop 3 is the center drop.

$(\Delta x)^{2}$ to the values 0.54 and 0.25 respectively.

The case $R e=15, C a=0.196=1.27 C a_{c}$ is shown in figures 10-11. As in the previous set of simulations, the first daughter drops are captured accurately at a rough mesh: for $\Delta x=\Delta y=\Delta z=1 / 64,1 / 96,1 / 128$, the daughter drop radius vs mother drop radius is $D / a=0.64$, which yields $54 \%$ of the critical volume. Table 7 shows drop data for different meshes, for the main drops disregarding small moons with $D / a<0.3$. The volumes of the main neck fragments again lie between $10 \%$ to $17 \%$ of the critical drop volume. Each main drop is typically followed by one or more small drops, or 'moons'. In the case of a rough mesh, a moon tends to take up more of the volume fraction than it would on a finer mesh, leaving less for the main drops. This explains the 

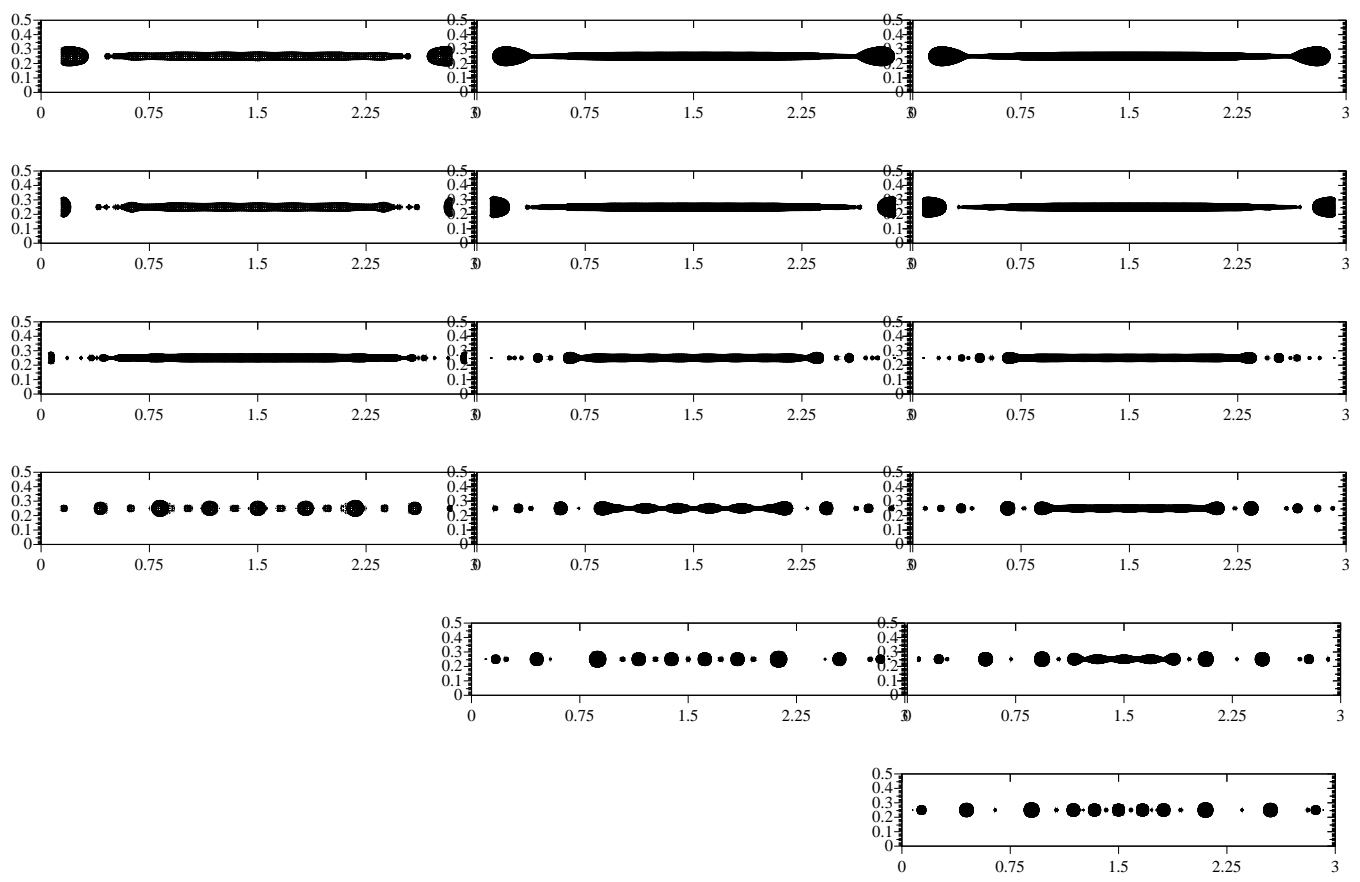

(a)

(b)

(c)

Fig. 10. $R e=15, C a=0.196=1.27 C a_{c}$, top view of computational domain $3 \times 0.5 \times 1$. Breakup sequence for (a) $\Delta x=1 / 64=\Delta y=\Delta z \cdot \mathrm{t}=20$ (drop 1 detached), 21, 24 (small moons), 28. (b) $\Delta x=1 / 96=\Delta y=\Delta z \cdot \mathrm{t}=20,21$ (drop 1 detached), 24 (drop 2 detached), 28, 31. (c) $\Delta x=1 / 128=\Delta y=\Delta z . \mathrm{t}=20,21$ (drop 1 has detached), 24 (small moons detach), 28 (drop 2 detached), 31 (drop 3 detached), 33 (drops 4, 5 and the center drop 6 detach). See table 7.

reduced number of main drops on the rough mesh. For the meshes, the total number of main drops is $7,10,11,8$, respectively. For the coarser meshes, 5 to 6 central drops break almost simultaneously, giving the appearance of capillary breakup of a jet. However, the finest mesh shows this to be repeated end-pinching of individual drops, in agreement with experimental observations of Marks (1998), that capillary breakup is observed for $C a / C a_{c}$ over 2. Comparing figures $10(\mathrm{~b})-11$, the simulations are similar up to $t=28 \mathrm{~s}$, and at this stage, some differences in the ends of the neck are observed. Based on the mesh refinement results of figure 9 , we expect the $1 / 160$ mesh result for the main neck fragments to be close to the correct sizes. This full-3D simulation required 7.5 hours $\mathrm{CPU}$ on 64 processors per 1s, totalling 10.6 days $\mathrm{CPU}$ for the results shown in the figure. 

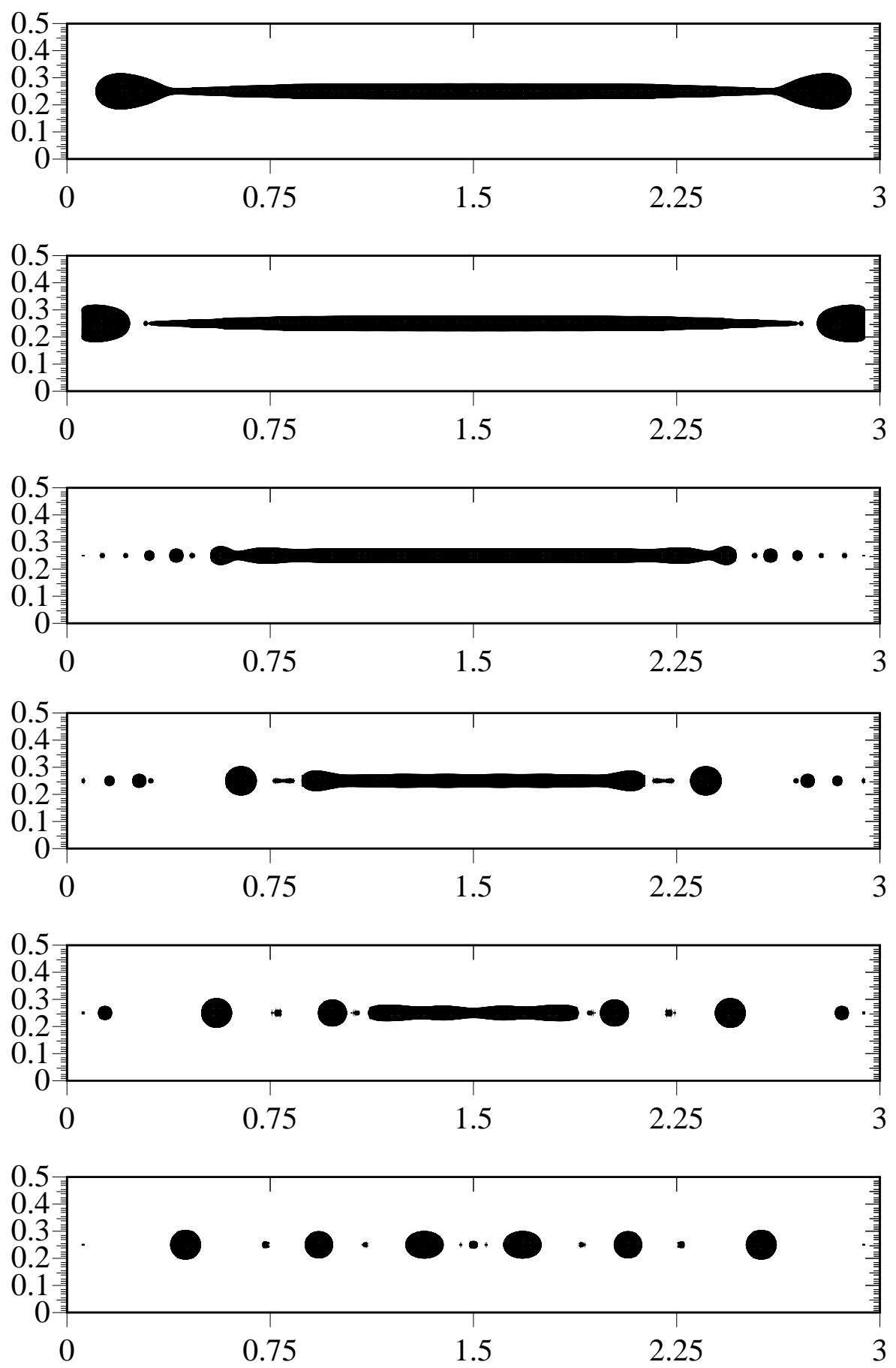

Fig. 11. $R e=15, C a=0.196=1.27 C a_{c}$, top view of computational domain $3 \times 0.5 \times 1$. Breakup sequence for $\Delta x=1 / 160=\Delta y=\Delta z . \mathrm{t}=20,21,24,28$, 31,34 s. See table 7 . 


\begin{tabular}{||c|cccc|cccc|cccc||}
\hline Drop & $\mathrm{D} / \mathrm{a}$ & & & & $C a_{D}$ & & & & $R e_{D}$ & & \\
$\Delta x$ & $1 / 64$ & $1 / 96$ & $1 / 128$ & $1 / 160$ & $1 / 64$ & $1 / 96$ & $1 / 128$ & $1 / 160$ & $1 / 64$ & $1 / 96$ & $1 / 128$ & $1 / 160$ \\
\hline 1 & 0.64 & 0.64 & 0.64 & 0.64 & 0.13 & 0.13 & 0.13 & 0.13 & 6.1 & 6.2 & 6.2 & 6.3 \\
2 & 0.34 & 0.35 & 0.38 & 0.43 & 0.07 & 0.07 & 0.07 & 0.08 & 1.7 & 1.9 & 2.1 & 2.8 \\
3 & - & 0.44 & 0.40 & 0.39 & - & 0.09 & 0.08 & 0.08 & - & 2.9 & 2.4 & 2.3 \\
4 & 0.45 & 0.36 & 0.35 & - & 0.09 & 0.07 & 0.07 & - & 3.0 & 1.9 & 1.8 & - \\
5 & 0.40 & 0.36 & 0.33 & 0.44 & 0.08 & 0.07 & 0.07 & 0.09 & 2.4 & 1.9 & 1.7 & 2.9 \\
6 & 0.40 & - & 0.33 & - & 0.08 & - & 0.06 & - & 2.4 & - & 1.6 & - \\
7 & 0.40 & 0.36 & 0.33 & 0.44 & 0.08 & 0.07 & 0.07 & 0.09 & 2.4 & 1.9 & 1.7 & 2.9 \\
8 & 0.45 & 0.36 & 0.35 & - & 0.09 & 0.07 & 0.07 & - & 3.0 & 1.9 & 1.8 & - \\
9 & - & 0.44 & 0.40 & 0.39 & - & 0.09 & 0.08 & 0.08 & - & 2.9 & 2.4 & 2.3 \\
10 & 0.34 & .35 & 0.38 & 0.43 & 0.07 & 0.07 & 0.07 & 0.08 & 1.7 & 1.9 & 2.1 & 2.8 \\
11 & 0.64 & 0.64 & 0.64 & 0.64 & 0.13 & 0.13 & 0.13 & 0.13 & 6.1 & 6.2 & 6.2 & 6.3 \\
\hline
\end{tabular}

Table 7

$R e=15, C a=0.196=1.27 C a_{c}$. Drop distribution. $3 \times 0.5 \times 1$, mesh $\Delta x=\Delta y=\Delta z$. 


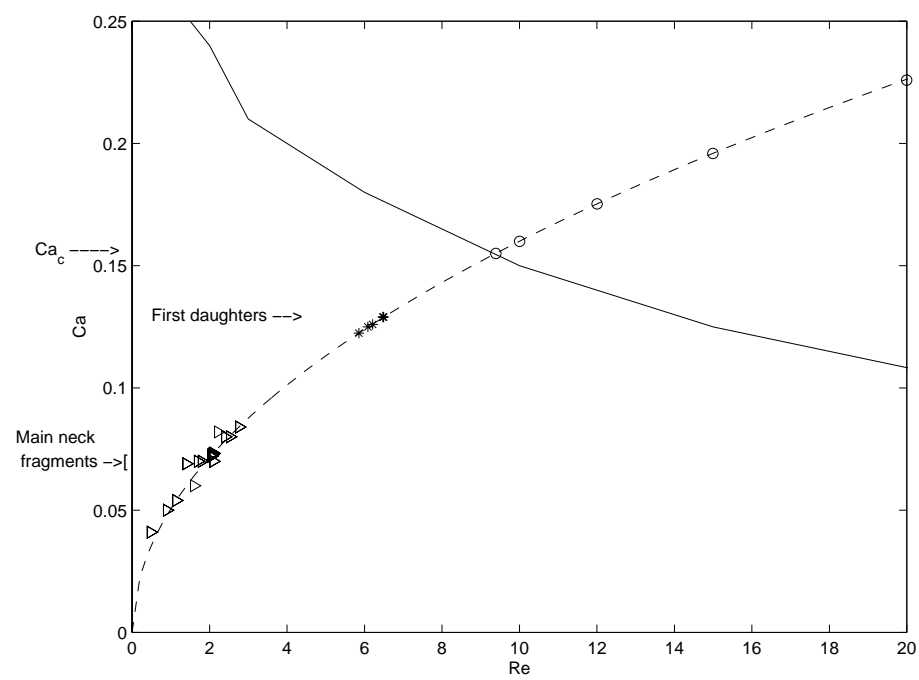

Fig. 12. $R e$ vs $C a$ for the critical curve (solid); $R e=K . C a^{2}$ (dashed), $K=391$, follows an experiment with fluid properties and flow strength fixed, while the radius of mother drop varies. Circles represent mother drop data. Asterisks denote first daughter drop data $R e_{D}$ vs $C a_{D}$. Triangles denote the rest of the fragments. Small fragments at the level of the discretized mesh are not included.

\section{$3 \quad$ Breakup with inertial effects}

In Renardy and Cristini (2000), we have investigated the size of the first daughter drops when the mother drop size is increased, and shown that they scale with the critical drop size. In this section, we extend the case $C a^{2}=391 R e$ treated in Renardy and Cristini (2000) to the full breakup simulation. Figure 12 shows the data on mother drops (o) for which we present numerical results. The first daughter drops $\left(^{*}\right)$ approach $54 \%$ of the critical volume (radius $D \approx 0.81 a_{c}$ ) as the mother drop size increases. Compared with Stokes flow (roughly $70 \%$ of volume, corresponding to $D \approx 0.9 a_{c}$ ), the effect of the additional parameter $R e$ is that the drops are smaller with inertia. The fragments from the neck $(\Delta)$ lie between $10 \%$ to $17 \%$ of the critical volume (radius $\approx 0.5 a_{c}$, to be contrasted to the value $0.64 a_{c}$ found by Cristini et al. (2001b) from experiments for drop-to-matrix viscosity ratio 0.1 ). The data are obtained from the three larger mother drops. Smaller fragments with $D / a<0.3$ occur in each numerical simulation and are not included in the plot because their volume fraction is small. These results indicate that the size of the large satellites produced from breakup events in the slender neck also scales at large capillary numbers with the critical size. This is supported by the simulations presented in the following of this section.

Figure 13 shows the velocity vector plot around a first daughter at $t=20 \mathrm{~s}$, showing that it is carried by the shear away from the neck. The neck is aligned at a slight angle to the imposed shear flow. The vectors are plotted at every 

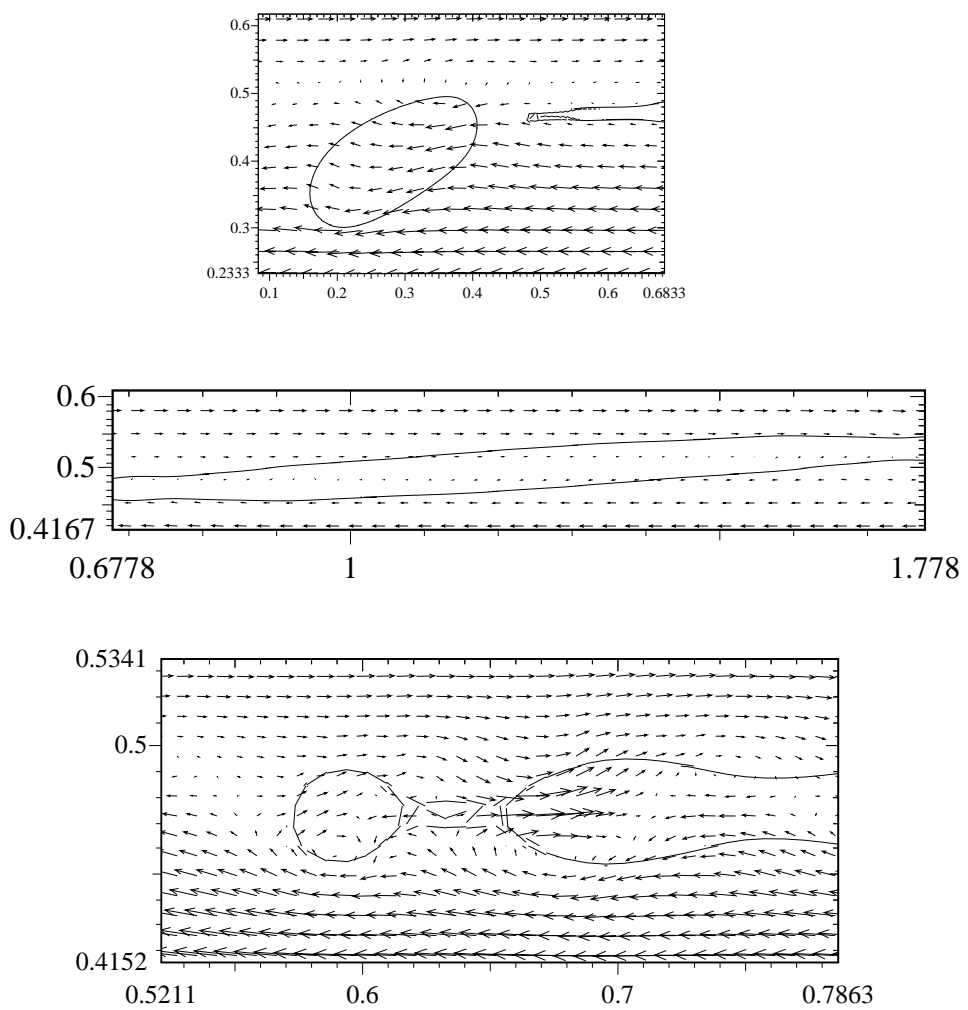

Fig. 13. $R e=12, C a=0.175=1.14 C a_{c}$, side view for $\mathrm{x}-\mathrm{z}$ plane at $\mathrm{y}=0.25$, computational domain $2.5 \times 0.5 \times 1$. mesh $\Delta x=\Delta y=\Delta z=1 / 128 . \mathrm{t}=20 \mathrm{~s}$, the neck is aligned at an angle to the imposed shear flow. Following this, the neck retracts to pinch-off.

four nodes. The next figure at a subsequent time shows that there is a retraction at the neck while the next drop is formed. The vectors here are plotted at every node.

The PacMan effect is sometimes observed during breakup: the drops are aligned at an angle to the imposed shear flow, so that they have different velocities and some of the drops spawned after the first daughters are seen to catch up to smaller drops and eat them. Figure 14 shows drops approaching each other for $\mathrm{t}=23,27 \mathrm{~s}$, at $R e=12$.

Figures 15-16 show the breakup simulations as the mother drop size increases. The neck contributes an increased volume fraction for roughly $0.4<C a_{D} / C a_{C}<0.6$. These drops are slightly smaller than for Stokes flow, for which experimental data in Section 4.6 of Marks (1998) put these in the range $0.5<C a_{D} / C a_{c}<0.75$. These Stokes flow results and the present work conclude that there are tiny drops that contribute relatively little volume fraction for $C a_{D} / C a_{c}<0.4$. 

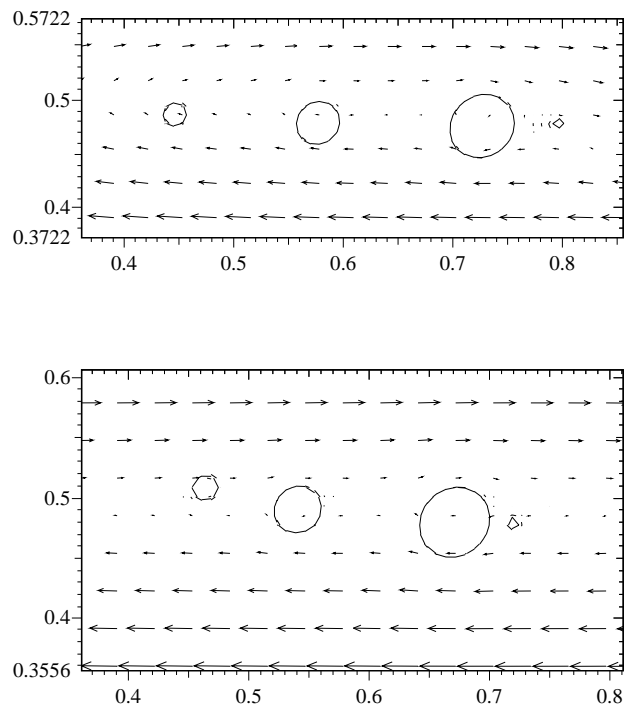

Fig. 14. $R e=12, C a=0.175=1.14 C a_{c}$, velocity vector plots for side-view in the $\mathrm{x}-\mathrm{z}$ plane through the center $\mathrm{y}=0.25$, computational domain $2.5 \times 0.5 \times 1$. mesh $\Delta x=\Delta y=\Delta z=1 / 128 . \mathrm{t}=23,27 \mathrm{~s}$.

At the instant when the first daughter drops pinch off, the neck is elongated to roughly its maximum length, and cylindrical in a central region, tapering off to pencil tips toward the ends. Table 8 tabulates the 'effective' capillary number for the cylindrical neck $C a_{n}$. This is calculated from the length $L$ of the neck (projected onto the x-axis) just after the first daughter drops detach, and the volume in the neck. An effective radius $r_{n}$ is calculated for this, assuming the neck is cylindrical, and of length $L$, or $\pi r_{n}^{2} L=(4 / 3) \pi a^{3}-2(4 / 3) \pi D^{3}$, so that

$$
r_{n}=\left[\frac{4 a^{3}\left(1-2\left(\frac{D}{a}\right)^{3}\right)}{3 L}\right]^{1 / 2}
$$

where $D$ is the first daughter drop radius, and

$$
C a_{n}=C a\left(r_{n} / a\right)
$$

At $R e=15$ (cf. table 7 ), the drops which result from the neck have capillary numbers $C a_{\text {Dneck }}$ between 0.08 and 0.09 , and if $C a_{\text {Dneck }}=F C a_{n}$, then $F$ ranges from 2.4 to 2.8. If the breakup were a capillary breakup of a viscous jet in a quiescent liquid as considered in Tjahjadi et al. (1992), then

$$
C a_{\text {Dneck }}=F C a_{n}, \quad F=2 .
$$

Here, however, we have repeated end-pinching, which produces larger drops. 


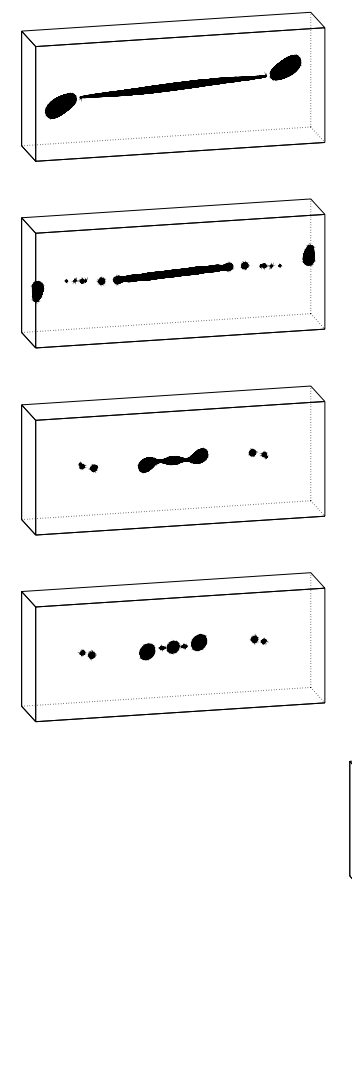

(a)
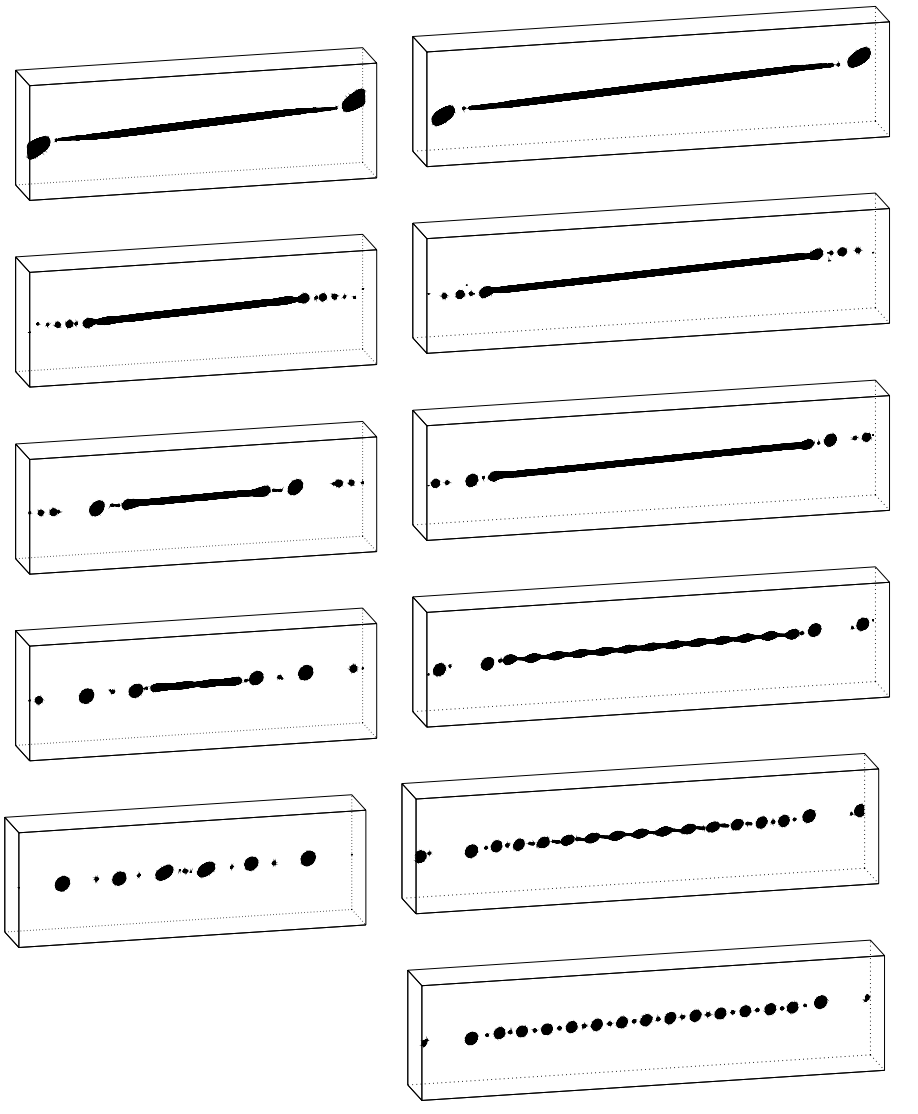

(b)

(c)

Fig. 15. (a) $R e=12, C a=0.175=1.14 C a_{c}$. Computational domain $2.5 \times 0.5 \times 1$. Mesh $1 / 192 . \mathrm{t}=20,23,28,29 \mathrm{~s}$. (b) $R e=15, C a=0.196=1.27 C a_{c}$. Computational domain $3 \times 0.5 \times 1$. Mesh $\Delta x=\Delta y=\Delta z=1 / 160 . \mathrm{t}=21,24,28,31$, 34. (c) $R e=20, C a=0.226=1.47 C a_{c}$. Computational domain $4 \times 0.5 \times 1$. Mesh $\Delta x=\Delta y=\Delta z=1 / 128 . \mathrm{t}=22$ (drop 1 has detached), 26 (moon $C a_{D}=.03, R e_{D}=0.3$, drop 2, moon $C a_{D}=0.02, R e_{D}=.15$, detach), 29 (drop 3 detached at 28s), 33 (drop 4), 34 (the rest of the drops detach), 35s.

For $R e=20, C a / C a_{c}=1.47$, figure $15(\mathrm{c})$ shows the evolution computed with a $1 / 128$ mesh. We expect this type of simultaneous breakup of the neck fragments at much higher capillary numbers. Based on mesh refinement studies mentioned in section 2, drops should end-pinch, resulting in fewer and larger drops. The numerical results obtained with a computational box of $4 \times 0.5 \times 1$ and $512 \times 64 \times 128$ grid yield 19 drops plus moons which account for a small percent of the mother drop volume. The values of $C a_{D}$ are: $0.13,0.08,0.08$, thirteen drops at $0.07,0.08,0.08,0.13$. The values of $V_{D} / V_{c}$ are 0.54 for the first daughters, and the rest lie between 0.10 and 0.16 , with many in the lower end. The moons have values of 0.04 or less. There are moons between every 


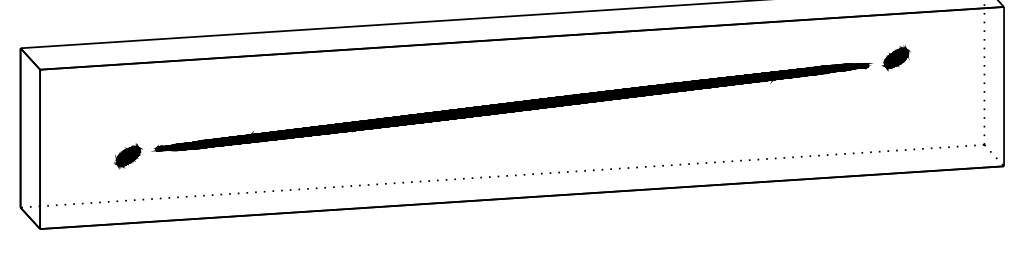

Fig. 16. $R e=37, C a=0.308=2 C a_{c}$. Computational domain $6 \times 0.5 \times 1$. Mesh $\Delta x=\Delta y=\Delta z=1 / 128 . \mathrm{t}=23 \mathrm{~s}$

\begin{tabular}{|c||ccccc||}
\hline$C a / C a_{c}$ & $C a_{D 1} / C a_{c}$ & $L$ & $r_{n}$ & $C a_{n} / C a_{c}$ & $L_{T}$ \\
\hline 1.14 & .81 & 1.57 & .021 & .19 & $2.18(20 \mathrm{~s})$ \\
1.27 & .82 & 2.40 & .023 & .23 & $2.79(20 \mathrm{~s})$ \\
1.47 & .81 & 3.18 & .023 & .27 & $3.54(21 \mathrm{~s})$ \\
\hline
\end{tabular}

Table 8

Capillary numbers for the mother $C a$ relative to critical $C a_{c}$, the first daughter drop $C a_{D 1}$, the effective neck radius $r_{n}$, the neck $C a_{n}$ relative to critical $C a_{c}$, neck length $L$ just after the first daughters pinch off, total length $L_{T}$ just before pinch-off, for the finest meshes presented in section 3 .

main drop. Each 1s shown in figure 15 (c) requires 13 to $14 \mathrm{CPU}$ hours on Origin 2200 with 8 processors. Figure 16 shows the simulation up to $23 \mathrm{~s}$ at twice the critical capillary number: $R e=37, C a=2 C a_{c}$. The neck is perfectly cylindrical for much of its length, and we would expect equation (7) to become more relevant.

Figure 17 shows the volume fraction relative to the mother drop volume, $V_{D} / V_{a}=\left(C a_{D} / C a_{c}\right)^{3} /\left(C a_{a} / C a_{c}\right)^{3}$, for each $C a_{D} / C a_{c}$. There are always tiny drops between the main fragments which have not been included in the graphs. The trend, as the mother drop size increases, is the growth in drops of size roughly half that of the mother drop. The first daughter drops remain as the largest drops, at roughly .8 of the mother drop volume, and there are no other drops of that magnitude in the simulations reported.

What is the scaling for the drops as the capillary number increases along the parabola shown in figure 12 ? The fate of the fragments is determined by the 


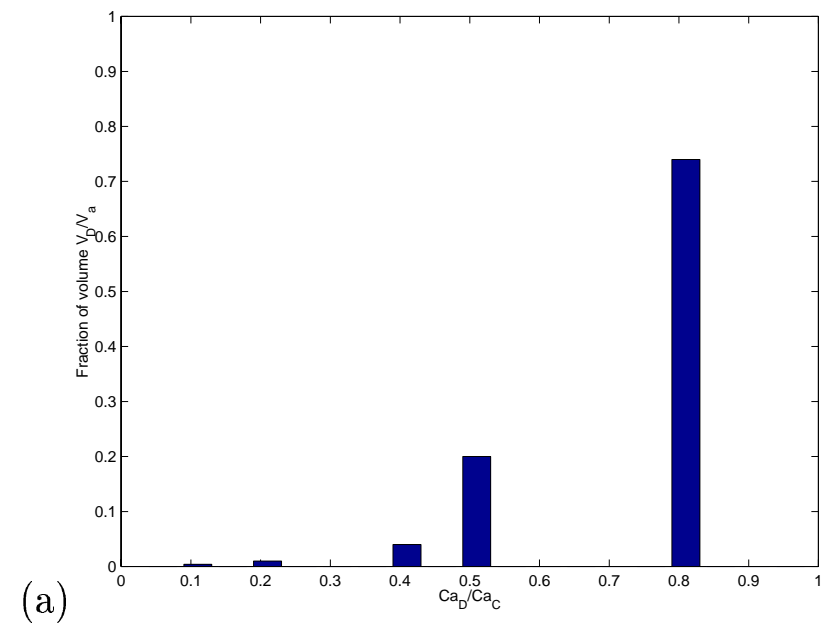

(a)

(b)

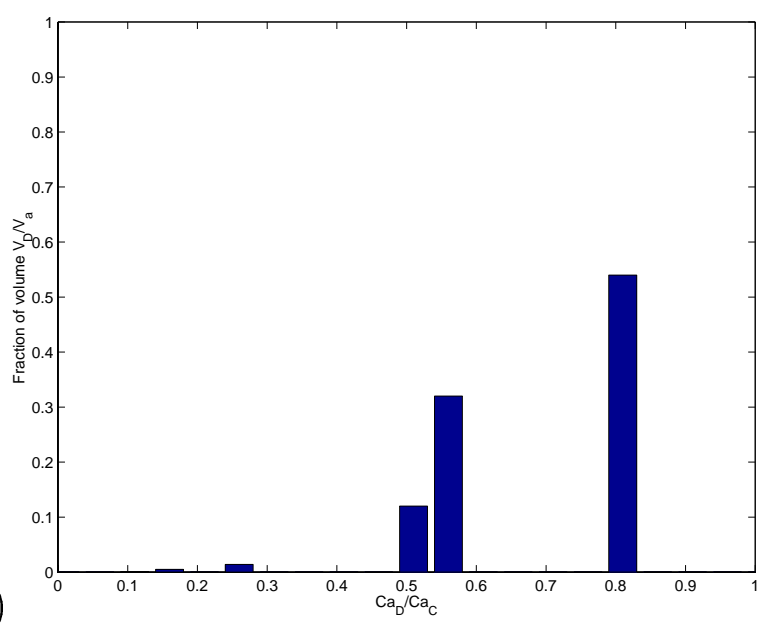

(c)

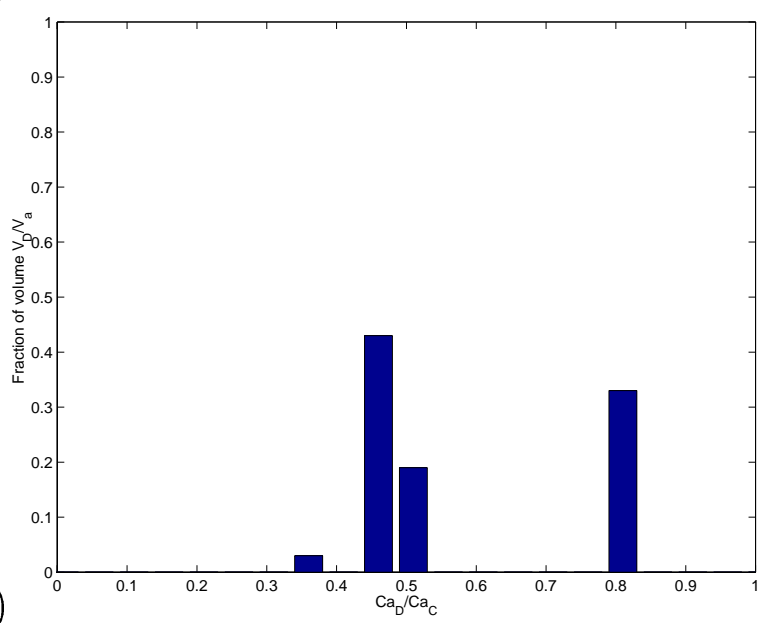

Fig. 17. Drop size distribution. (a) $R e=12, C a=0.14 C a_{c}, 2.5 \times 0.5 \times 1$, mesh $\Delta x=1 / 192=\Delta y=\Delta z$. (b) $R e=15, C a=1.27 C a_{c}, 3 \times 0.5 \times 1$, mesh $\Delta x=1 / 160=\Delta y=\Delta z$. (c) $R e=20, C a=1.47 C a_{c}, 4 \times 0.5 \times 1$, mesh $\Delta x=1 / 128=\Delta y=\Delta z$. This excludes moons which have $V_{D} / V_{a} \approx .002$. There are moons between every main drop and account for a few percentage of the mother drop size. 
first daughter drops. These scale with the critical volume (cf table 8). The neck radius determines the size of the subsequent drops because they are the same order of magnitude. As the capillary number increases to infinity, the ratio of daughter drop radii to mother drop radius decreases to zero, $D / a \rightarrow 0$ and equation (5) shows that the effective neck radius satisfies

$$
r_{n} \sim L^{-1 / 2} a^{3 / 2}
$$

The capillary numbers covered in table 8 are not sufficiently large for this scaling to apply, because the ratio $D / a$ is not negligible. Therefore, in our regime, $r_{n}$ is influenced by the two competing effects in equation (5): $L$ increases with increasing $C a$, and the numerator $1-2(D / a)^{3}$ also increases.

For large capillary numbers, the effect of surface tension is initially small and the drop stretches following the simple shear flow, elongates, until eventually, the cross-section of the drop evolves to a circular shape, length scales are reached at which surface tension becomes important, and pinching begins. This pinching begins when an effective radius is approximately the critical radius:

$$
r_{e} \approx r_{c}, \quad r_{c}=a \frac{C a_{c}}{C a}
$$

This effective radius might be interpreted either as the effective neck radius for an extremely elongated case, but more appropriately for our simulations, as an average radius for a less elongated shape, where $\pi r_{e}^{2} L_{T}$ equals the original drop volume, with $L_{T}$ being the total length. When pinch-off occurs, the total length of the drop is of order

$$
L_{T} \sim r_{c}^{-2} a^{3} \sim a\left(\frac{C a}{C a_{c}}\right)^{2} .
$$

Note that along the parabola of figure $12, R e=K . C a^{2}$, and equation (10) gives

$$
L_{T} \sim R e
$$

From table 8, we see that the ratios of $L_{T}$ are consistent with the scaling of equation (11). For instance, $L_{T, R e=12} / L_{T, R e=15}$ with within $2 \%$ of the ratio of the Reynolds numbers $12 / 15 . L_{T, R e=15} / L_{T, R e=20}$ is within $5 \%$ of the ratio of the Reynolds numbers 15/20.

The scaling for the first daughter drops and neck fragments aid in interpreting the experimental data of Section 4.6 of Marks (1998), albeit the data are for 
Stokes flow, for which the daughter drops are slightly larger than at higher Reynolds numbers. His figure 4.6.1.a gives five sample histograms of daughter drop sizes, placed in four bins. The $1.0 \mathrm{bin}, 0.75 \mathrm{bin}, 0.50 \mathrm{bin}$ and $0.25 \mathrm{bin}$ represent $0.75<C a_{D} / C a_{c}, 0.50<C a_{D} / C a_{c}<0.75,0.25<C a_{D} / C a_{c}<0.50$, $C a_{D} / C a_{c}<0.25$, respectively. The first daughter drops fall into the 1.0 bin. The neck fragments mostly fall into the $0.75 \mathrm{Bin}$, while tiny drops fall into the other two bins. Beyond the production of the two largest daughters, no other drops of this order of magnitude are produced because the rest of the drops come from the elongated neck. The trend, therefore, is that as the mother capillary number increases, the volume fraction in the 0.75 bin increases and eventually dominates. The histogram at $K_{i}=1.0$ denotes the fact that just above criticality, most of the drop goes into the first daughter drops in the 1.0 bin. Our results at Reynolds number order 10 suggest that just two daughter drops fall into the 1.0 bin and the neck fragments fall into the 0.75 bin. The moons fall into the smaller bins. We predict using just this, that when $K_{i}=$ $C a / C a_{c}=2$, the volume fraction of the neck is 0.86 , and when $K_{i}=6.1$, it is 0.995. This is a close prediction of the two histograms in his figure.

The drop size distribution for large capillary numbers which we predict is observed in the experimental results of Cristini et al. (2001b) for viscosity ratio $\lambda=0.1$ and Stokes flow. They show that the total number of main fragments $N$ scales as

$$
N \sim(k / \alpha)^{3}
$$

where $k=C a / C a_{c}$ and $\alpha$ is the fragment radius/critical radius. The scaling can be easily obtained from conservation of volume and from the observation that the fragment radius tends to scale with the critical radius at large enough $C a$. In our simulations, $\alpha \approx 0.5$, while in the experiments, $\alpha \approx 0.64$. The difference is plausible, considering the difference in Reynolds number and viscosity ratio.

\section{References}

Blawzdziewicz, J., Cristini, V., Loewenberg, M., 2000. Critical behavior of drops in linear flows: I. phenomenological theory. Phys. fluids, submitted.

Cristini, V., 2000. Drop dynamics in viscous flow. PhD thesis, Yale University.

Cristini, V., Blawzdziewicz, J., Loewenberg, M., 1998. Drop breakup in threedimensional viscous flows. Phys. fluids 10(8), 1781-1783.

Cristini, V., Blawzdziewicz, J., Loewenberg, M., 2001a. An adaptive mesh algorithm for evolving surfaces: simulations of drop breakup and coalescence. J. Comp. Phys. $\mathrm{x}$, in press. 
Cristini, V., Guido, S., Alfani, A., Blawzdziewicz, J., Loewenberg, M., 2001b. Drop breakup in shear flow. J. Fluid Mech., submitted.

Eggers, J., 1995. Theory of drop formation. Phys. Fluids 7, 941-953.

Gueyffier, D., Li, J., Nadim, A., Scardovell, R., Zaleski, S., 1999. Volumeof-fluid interface tracking and smoothed surface stress methods for threedimensional flows. J. Comp. Phys. 152, 423-456.

Guido, S., Villone, M., 1998. Three-dimensional shape of a drop under simple shear flow. J. Rheol. 42, 395-415.

Kennedy, M. R., Pozrikidis, C., Skalak, R., 1994. Motion and deformation of liquid drops and the rheology of dilute emulsions in simple flow. Comput. \& Fluids 23, 251.

Lafaurie, B., Nardone, C., Scardovelli, R., Zaleski, S., Zanetti, G., 1994. Modelling merging and fragmentation in multiphase flows with SURFER. J. Comp. Phys. 113, 134-147.

Li, J., 1995. Calcul d'Interface Affine par Morceaux (Piecewise Linear Interface Calculation). C. R. Acad. Sci. Paris t.320 série IIb, 391-396.

Li, J., Renardy, Y., 1999. Direct simulation of unsteady axisymmetric coreannular flow with high viscosity ratio. J. Fluid Mech. 391, 123-149.

Li, J., Renardy, Y., Renardy, M., 1998. A numerical study of periodic disturbances on two-layer Couette flow. Phys. Fluids 10, 3056-3071.

Li, J., Renardy, Y., Renardy, M., 2000. Numerical simulation of breakup of a viscous drop in simple shear flow through a volume-of-fluid method. Phys. Fluids 12(2), 269-282.

Lister, J. R., Stone, H. A., 1998. Capillary breakup of a viscous thread surrounded by another viscous fluid. Phys. Fluids 10, 2758-2764.

Marks, C. R., 1998. Drop breakup and deformation in sudden onset strong flows. Ph.D. thesis, University of Maryland at College Park.

Papageorgiou, D. T., 1995. On the breakup of viscous liquid threads. Phys. Fluids 7, 1529-1544.

Rayleigh, L., 1900. Scientific Papers. Cambridge Univ. Press.

Renardy, Y., Cristini, V., 2000. Scalings for fragments produced from drop breakup in shear flow with inertia. Phys. Fluids submitted, - .

Renardy, Y., Cristini, V., 2001. Effect of inertia on drop breakup under shear. Phys. Fluids Jan., -.

Renardy, Y., Li, J., 2000. Parallelized simulations of two-fluid dispersions. SIAM News in 'Applications on Advanced Architecture Computers', G. Astfalk ed., December, 1.

Renardy, Y., Renardy, M., Cristini, V., 2000. A new volume-of-fluid formulation for surfactants and simulations of drop deformation under shear at a low viscosity ratio. Eur. J. Mech. B/ Fluids submitted.

Scardovelli, R., Zaleski, S., 1999. Direct numerical simulation of free surface and interfacial flow. Ann. Rev. Fluid Mech. 31, 567-604.

Tjahjadi, M., Stone, H. A., Ottino, J. M., 1992. Satellite and subsatellite formation in capillary breakup. J. Fluid Mech. 243, 297-317.

Utracki, L. A., Shi, Z. H., 1992. Development of polymer blend morphology 
during compounding in a twin-screw extruder. Part I: droplet dispersion and coalescence - a review. Part II: Theoretical derivations. Part III: experimental procedure and preliminary results. Polymer Eng. Sci. 32:24, 1824-1856. 